\title{
Review of Atom Probe Tomography Applications for Semiconductor materials.
}

\author{
L. Renaud ${ }^{1 *}$, I. Martin ${ }^{1}$, B. Sallé ${ }^{1}$, R. Benbalagh ${ }^{1}$, A. Davis ${ }^{2}$ and M. Schuhmacher ${ }^{1}$. \\ ${ }^{1}$ CAMECA 29 Quai des Grésillons, 92622 Gennevilliers Cedex - France. \\ ${ }^{2}$ CAMECA Inc, 4301 Garrity Blvd, \#201, Nampa, ID 83687 \\ *corresponding author: renaud@cameca.com
}

In 3D Atom Probe Tomography, the LASER evaporation technique has been shown to be well suited for the analysis of many materials including semi-conductor compounds.

Moreover, with the Focused Ion Beam (FIB) techniques (protective cap layer, lift-out, low energy Ga beam milling....), samples ready for 3D-AP analysis can be extracted and prepared from a specific location at tenths of $\mu \mathrm{m}$ scale in btoh blanket and patterned wafers.

In this study, we use the technique of femtosecond LASER evaporation, providing 3 different wavelengths in order to generate the field for atom evaporation. This flexible configuration ensures optimized conditions depending on the nature of the sample and nano-scale structure. Indeed, under these conditions, field-effect atom evaporation is completed with near $100 \%$ ionization yield, and atoms are projected onto a position sensitive detector (Advance DelayLine type) with detection efficiency approaching $60 \%$ even in case of multiple hits. Each particle reaching the detector is identified by time of flight mass spectrometry. The combination of high atom ionization yield and high detection efficiency ensures detection limits of tenths of atomic percent at ultra high spatial resolution. For example, in the case of a dopant concentration of $0.2 \%, 60$ atoms over the 100 atoms present in a $10 \mathrm{~nm}^{3}$ volume are detected.. And for each detector "hit", the position information at the sample surface is determined with a lateral resolution of $<0.5 \mathrm{~nm}$ and a depth resolution of $<0.3 \mathrm{~nm}$.

This paper will review the latest instrumental developments in Atom Probe Tomography and how they have positively impacted the application field for semiconductors. Results obtained for dopant profiling, III-V layer homogeneity, high-k thin insulating layers, ... will be presented. In conclusion, potentials and limits of the 3D-AP technique for nano-scale materials analysis will be discussed. 